\title{
Intervalli di riferimento comuni? Utili? Possibili? Necessari?
}

\author{
Common reference intervals? Useful? Possible? Necessary?
}

\author{
Romolo M. Dorizzi • Paolo Maltoni
}

Ricevuto: 9 marzo 2011 / Accettato: 11 marzo 2011

(C) Springer 2011

Summary The theory of the reference interval has been well-defined by authoritative professional institutions but its practical application in most clinical laboratories is far from optimal. Laboratories often use different reference intervals without any valid reason, such as variations in analytical methodology or the population served. The adoption of common reference intervals has become a matter of importance because: (1) several common decision points which are not determined or validated in individual laboratories, e.g. glucose and lipid measurements, have already been widely accepted; (2) common reference intervals have been developed in Scandinavia by an international clinical chemistry organisation

Non basta sapere, si deve anche applicare; non è abbastanza volere, si deve anche fare. Imparare a dominare è facile, ma a governare è difficile. Senza fretta, ma senza sosta. Johann Wolfgang Goethe 1749-1832

R.M. Dorizzi · P. Maltoni

Laboratorio Unico di Area Vasta Romagna,

Pievesestina di Cesena (FC)

\section{R.M. Dorizzi (局)}

U.O.C. CoreLab Laboratorio Unico di Area Vasta Romagna,

Piazzale della Liberazione n. 60,

47522 Pievesestina di Cesena (FC)

Tel.: +39-0547-394809,

Fax: +39-045-74431187

e-mail: rdorizzi@ausl-cesena.emr.it
(Nordic Reference Interval Project, NORIP) and are being developed in Oceania; (3) the approach has been discussed favourably at international meetings; and (4) assay standardisation has substantially advanced (e.g. in the European Union). In 1998 the NORIP programme was started with the participation of 102 Nordic clinical biochemistry laboratories which collected blood samples from at least 25 healthy reference individuals evenly distributed for gender and age, and determined 25 of the most commonly requested serum/plasma components for each reference individual. Analytical and methodological data and data describing the reference individuals were submitted to a central database for evaluation and calculation of reference intervals intended for common use in the Nordic countries. Today, common reference intervals are used by almost $100 \%$ of laboratories for some analytes (e.g. iron, bilirubin, urate, magnesium). In 2005/06 the UK Department of Health set up a national Action Learning Programme as a key part of its Modernising Pathology Programme to enable people working in NHS pathology services to tackle issues and problems they identify locally as a priority for action. The Department funded six action learning sets to work on priority areas in pathology: workforce, standardisation, networks, primary care and protocol-guided investigations. The first phase was a success and was followed by a second phase with 16 topics. Work on the learning set for harmonise of reference intervals was carried out in the West Midlands. The set took a pragmatic approach to harmonising the variations between laboratories. Different practices were identified and were examined to see whether reasons for the differ- 
ences were technical, scientific or clinical (e.g. differences in equipment and reagents or in the population served). The variations at the top and bottom were then considered, and a proposal based on consensus was developed. From 2007, the Pathology Harmony Initiative started to look at the harmonisation within the clinical laboratory. Several areas were identified where it was felt that there was potential for harmonisation. Several topics (e.g. units of measurement, reference intervals, test name harmonisation) have been investigated. All these experiences demonstrate that harmonisation of the reference intervals for several analytes should at last also be considered in Italy.

Key words Common reference interval - NORIP . Pathology Harmony · Quality control

\section{Introduzione}

Qualche anno fa Ferruccio Ceriotti, probabilmente il più importante cultore della materia nel nostro paese, ha sintetizzato in alcuni articoli molto completi e interessanti $[1,2]$ l'esperienza acquisita nell'aggiornamento dello standard Clinical and Laboratory Standards Institute (CLSI) C28A3 [3]. Citiamo con lui un classico di mezzo secolo fa, opera di Albert John Schneider [4]. La prima funzione del medico è quella di decidere se un paziente appartiene ad una categoria mal definita di individui sani o alla classe generale di individui malati. Il medico non prende questa decisione sulla base della impressione generale ma "micronizza" un paziente in modo che non è più una persona ma una associazione nel tempo e nello spazio variamente assortita di attributi e caratteristiche. Ognuno di questi attributi è normale o non, singolarmente o associato agli altri. Se troviamo una determinata associazione di attributi anomali, il paziente è poi riassemblato come malato "di una malattia nota" $o$ diventa il primo malato "di una nuova malattia". Se non si trova nessuna associazione, il soggetto "rinasce", almeno per il momento, come sano.

\section{Cresce la consapevolezza}

Non si può che essere d'accordo nell' attirare l'attenzione verso le conclusioni del pediatra americano: la medicina che pratichiamo sarà davvero una scienza, non solo se comprendiamo le relazioni strutturali, funzionali e chimiche in azione nell'individuo, ma se comprendiamo le basi delle nostre misure e dei nostri confronti. I principali fattori limitanti dei confronti "validi" sono la grossolanità della base dei confronti, la pigrizia con cui affrontiamo questi aspetti e lo scarso/nullo impiego dei corretti strumenti statistici e organizzativi per affrontare la cosiddetta fase post-analtica.

C'è da restare sbalorditi nel rilevare quanto impegno sia profuso nella messa a punto di metodi analitici sempre più sensibili, specifici e veloci per produrre dei risultati corredati poi da intervalli di riferimento del tutto inadeguati. Ceriotti presenta un caso, drammaticamente evidente, relativo agli intervalli di riferimento adottati da un gruppo di 93 laboratori italiani, che utilizzavano lo stesso metodo International Federation of Clinical Chemistry (IFCC) con piridossal fosfato per la misura dell'attività catalitica dell'alanina amminotransferasi (ALT): almeno la metà dei laboratori non forniva intervalli di riferimento distinti per sesso e il limite superiore variava da 30 U/L a $72 \mathrm{U} / \mathrm{L}$, sia per i maschi sia per le femmine. Non tutti gli utilizzatori di strumenti Dade-Behring ${ }^{\circledR} \mathrm{e}$ Vitros $^{\circledR}$, che fornivano risultati decisamente superiori al resto del gruppo, utilizzavano gli intervalli di riferimento proposti da queste aziende (nel caso del Vitros ${ }^{\circledR}$ meno della metà). È stato, inoltre, segnalato frequentemente in passato e anche di recente, che laboratori che dichiaravano di aver adottato lo stesso intervallo di riferimento suggerito dal produttore, avevano in realtà intervalli per il TSH e per il testosterone diversi [5, 6]. Al contrario, intervalli derivati o dal produttore o calcolati da laboratorio, non differivano per un ampio pannello di analiti (potassio, calcio, magnesio, TSH, emoglobina, piastrine e PTT) [7].

Spesso per il clinico e per il paziente non è facile confrontare risultati ottenuti in diversi laboratori (lo stesso valore può essere considerato fisiologico o patologico a seconda del laboratorio in cui è stato ottenuto) e diventa impossibile approfittare di ciò che la tecnologia informatica rende possibile: combinare risultati ottenuti da diversi laboratori in una banca dati comune. Lo stesso CLSI riconosce, però, che l'effettiva definizione degli intervalli di riferimento fatta sulla popolazione afferente al laboratorio risulta proibitiva, in termini economici e di tempo, anche solo per un singolo analita e diventa impossibile per le centinaia di analiti misurati da un laboratorio, con metodi e strumenti che cambiano frequentemente negli anni.

Una possibile soluzione per superare questa situazione è l'elaborazione di intervalli di riferimento "comuni". Già negli anni ottanta la Commissione Regionale CQ Interlab della Regione Emilia Romagna aveva trovato "una situazione che obbliga a profonde e importanti riflessioni sulle iniziative da intraprendere ... per la chimica clinica il $90 \%$ degli intervalli di riferimento proviene da indicazioni riportate nelle metodiche elo riprese dalla letteratura". Il commento era preoccupato e preoccupante: "Le informazioni ricavate del programma di 
controllo hanno poi confermato che molti I.R. non siano in sintonia con le prestazioni del metodo. In questa condizione un risultato "concordante" in termini numerici può trasformarsi in un risultato significativamente non coincidente quando normalizzato" [8].

In quegli stessi anni in molti paesi si cominciava a cercare rimedi alla "babelizzazione" degli intervalli di riferimento e al dato di fatto che intervalli diversi erano adottati da laboratori posti nella stessa area e che usavano lo stesso analizzatore, mentre intervalli di riferimento identici erano adottati da laboratori che operavano anche in continenti diversi e che usavano analizzatori diversi con un bias molto evidente.

\section{L'esperienza scandinava}

Nella primavera del 1998 si costituiva in Scandinavia un gruppo di lavoro con l'obiettivo di affrontare in modo strutturato il problema, costituito da Peter Felding e Per Hyltoft Petersen dalla Danimarca, Leifur Franzson dall'Islanda, Veli Kairisto dalla Finlandia, Pal Rustad dalla Norvegia, Gunnar Skude, Kristoffer Hellsing e Per Simonsson dalla Svezia ed esperti del calibro di Adam Uldall, Heidi Steensland, Ari Lahti, Gunnar Nordin e Minna Loikkanen. L'obiettivo era quello di estendere all'intera Scandinavia il progetto di armonizzare gli intervalli di riferimento (dei principali parametri di biochimica e di ematologia) in corso nell'area di Copenhagen.

Una volta definiti in modo molto preciso i criteri di reclutamento e di trattamento dei campioni, 102 laboratori scandinavi hanno selezionato almeno 25 soggetti distribuiti omogeneamente per quanto riguarda età e sesso. Sono stati selezionati oltre 3.000 soggetti e oltre 160.000 campioni di siero; in circa il $60 \%$ di questi soggetti, provenienti soprattutto da Finlandia e Svezia, è stato raccolto anche materiale biologico per la definizione degli intervalli di riferimento in ematologia [9]. Un'aliquota era analizzata dal singolo laboratorio insieme a del materiale di controllo per stimare il bias del metodo, mentre 7 aliquote di siero e 2 di plasma erano inviate a una biobanca centrale.

Il lavoro, come si vede, è stato imponente e nel 2004 un fascicolo speciale dello Scandinavian Journal of Clinical Laboratory Investigation ne ha pubblicato i risultati per i principali esami di biochimica, enzimologia ed ematologia. Questi sono poi diventati disponibili in modo più completo anche sul portale del progetto [10]. Si può rilevare che gli intervalli, nei diversi paesi della Scandinavia, sono abbastanza comparabili e sul portale del Nordic Reference Interval Project (NORIP) è possibile seguire il dibattito che ha accompagnato l'introduzione dei nuovi intervalli nei diversi paesi. Nel giugno 2010, Pal Rustad ha presentato al XXXII Congress in Medical Biochemistry di Oslo la relazione: "The Nordic Reference Interval Project 2000. Concept and consequences" che ha rappresentato una buona occasione per fare un bilancio dei primi 10 anni di attività [11]. È interessante notare che, in generale, Danimarca e Finlandia sono i paesi che hanno applicato più estesamente $\mathrm{i}$ nuovi intervalli $\mathrm{e}$ che per alcuni parametri (ferro, bilirubina, urato, magnesio) l'adesione è prossima al $100 \%$ nei quattro paesi e per altri, come creatinina, urea e fosfato, è molto alta. Alcune proposte di intervalli sono state accolte molto meno favorevolmente. La proposta che ha avuto minor successo è quella relativa al potassio, ma, in effetti, il valore di 4,4 mmol/L come limite superiore non appare immediatamente convincente.

\section{L'esperienza anglosassone}

Nei primi anni 2000 anche in Oceania si è aperto il dibattito sugli intervalli di riferimento comuni. Gli argomenti principali a favore della loro adozione erano:

1. i limiti decisionali comuni erano già impiegati diffusamente per molti dosaggi senza che il singolo laboratorio li abbia validati (tra i più consolidati quelli relativi a glucosio, lipidi, emoglobina glicata, PCR ad alta sensibilità, troponina $\mathrm{T} \mathrm{hs}$ );

2. gli intervalli di riferimento comuni erano già impiegati da molti anni nell'area di Auckland e in Scandinavia (NORIP);

3. la proposta era già stata discussa con favore in convegni internazionali;

4. la standardizzazione di molti metodi era progredita marcatamente negli ultimi anni.

Tra gli ostacoli che sono stati rilevati anche in Oceania vi sono le grosse differenze tra i metodi immunometrici e le differenze in alcuni parametri tra razze diverse. È interessante notare che il primo aspetto affrontato è stato la standardizzazione del referto per quanto riguarda: denominazione dell'esame, unità di misura, numero di decimali ed eventuale arrotondamento [12].

Nel 2007 è stato condotto un audit per verificare quanto questo processo fosse progredito. Vi sono state delle rilevanti esperienze regionali molto sviluppate come The Auckland Region QA Group (ARQAG) che comprende dieci laboratori nella parte settentrionale della North Island della Nuova Zelanda, che stanno impiegando su base continuativa, dal 1976, intervalli di riferimento comuni e, quando necessario, li stanno aggiornando. Questo progetto ha avuto successo ed è in fase di progettazione la sua estensione a tutta l'Oceania e oltre [13]. 
Tabella 1 Intervalli di riferimento proposti dal programma Pathology Harmonization e intervalli usati dal Laboratorio Unico di AVR

\begin{tabular}{|c|c|c|c|c|c|c|c|c|c|}
\hline \multirow[b]{2}{*}{ Analita } & \multicolumn{4}{|c|}{$\begin{array}{c}\text { Intervalli di riferimento proposti } \\
\text { da Pathology Harmonization (adulti) }\end{array}$} & \multicolumn{5}{|c|}{ Intervalli di riferimento usati in AVR } \\
\hline & U.M. & inf. & sup. & note & U.M. & fatt. conv. & inf. & sup. & note \\
\hline Sodio & $\mathrm{mmol} / \mathrm{L}$ & 133 & 146 & & & & 136 & 145 & \\
\hline Potassio & $\mathrm{mmol} / \mathrm{L}$ & 3,5 & 5,3 & & & & 3,5 & 5,1 & \\
\hline Urea & $\mathrm{mmol} / \mathrm{L}$ & 2,5 & 7,8 & & $\mathrm{mg} / \mathrm{dL}$ & 6,006 & & $<8,3$ & Diviso in 2 fasce d'età \\
\hline Cloro & $\mathrm{mmol} / \mathrm{L}$ & 95 & 108 & & & & 98 & 107 & \\
\hline Bicarbonato & $\mathrm{mmol} / \mathrm{L}$ & 22 & 29 & & & & 24 & 28 & \\
\hline Fosfato & $\mathrm{mmol} / \mathrm{L}$ & 0,8 & 1,5 & & $\mathrm{mg} / \mathrm{dL}$ & 3,1 & 0,87 & 1,45 & $\begin{array}{l}\text { Diviso per fasce d'età } \\
\text { e per sesso }\end{array}$ \\
\hline Magnesio & $\mathrm{mmol} / \mathrm{L}$ & 0,7 & 1 & & $\mathrm{mg} / \mathrm{dL}$ & 2,43 & 0,66 & 1,07 & \\
\hline Albumina & $\mathrm{g} / \mathrm{L}$ & 35 & 50 & & & & 35 & 52 & \\
\hline Proteine Totali & $\mathrm{g} / \mathrm{L}$ & 60 & 80 & & & & 66 & 87 & \\
\hline Osmolalità & $\mathrm{mmol} / \mathrm{kg}$ & 275 & 295 & & & & 275 & 300 & \\
\hline \multirow[t]{2}{*}{ Fosfatasi Alcalina (ALP) } & $\mathrm{U} / \mathrm{L}$ & 30 & 130 & $\mathrm{M}$ & & & 40 & 129 & $\begin{array}{l}\text { Diviso per fasce d'età } \\
\text { e per sesso }\end{array}$ \\
\hline & & & & $\mathrm{F}$ & & & 35 & 104 & \\
\hline \multirow[t]{2}{*}{ Creatina Kinasi (CK) } & $\mathrm{U} / \mathrm{L}$ & 40 & 320 & M & & & 39 & 308 & \\
\hline & & 25 & 200 & $\mathrm{~F}$ & & & 26 & 192 & \\
\hline Bilirubina (totale) & $\mu \mathrm{mol} / \mathrm{L}$ & & $<21$ & & $\mathrm{mg} / \mathrm{dL}$ & 0,0585 & & $<20,5$ & Diviso per fasce d'età \\
\hline Calcio & $\mathrm{mmol} / \mathrm{L}$ & 2,2 & 2,6 & & $\mathrm{mg} / \mathrm{dL}$ & 4,01 & 2,1 & 2,5 & \\
\hline \multirow[t]{2}{*}{ Urato } & $\mu \mathrm{mol} / \mathrm{L}$ & 200 & 430 & $\mathrm{M}$ & $\mathrm{mg} / \mathrm{dL}$ & 59,5 & 202 & 416 & \\
\hline & & 140 & 360 & $\mathrm{~F}$ & & & 143 & 339 & \\
\hline Carbamazepina & $\mathrm{mg} / \mathrm{L}$ & 4 & 12 & & & & 8 & 12 & \\
\hline Fenobarbitone & $\mathrm{mg} / \mathrm{L}$ & 10 & 40 & & & & 15 & 40 & \\
\hline Fenitoina & $\mathrm{mg} / \mathrm{L}$ & 5 & 20 & & & & 10 & 20 & \\
\hline Teofillina & $\mathrm{mg} / \mathrm{L}$ & 10 & 20 & & & & 10 & 20 & \\
\hline Valproato & $\mathrm{mg} / \mathrm{L}$ & & & & & & 50 & 100 & \\
\hline Paracetamolo & $\mathrm{mg} / \mathrm{L}$ & & & & & & 10 & 30 & \\
\hline Salicilato & $\mathrm{mg} / \mathrm{L}$ & & & & & & 150 & 300 & \\
\hline Metotrexate & $\mu \mathrm{mol} / \mathrm{L}$ & & & & & & & $>5$ & \\
\hline Litio & $\mathrm{mmol} / \mathrm{L}$ & 0,4 & 1 & & & & 0,6 & 1,2 & \\
\hline Digossina & $\mu g / L$ & 0,5 & 1 & & & & 0,5 & 1 & \\
\hline Tacrolimus & $\mu \mathrm{g} / \mathrm{L}$ & & & & & & 5 & 20 & \\
\hline $25 \mathrm{OH}$ Vitamina D & $\mathrm{nmol} / \mathrm{L}$ & & & & $\mu \mathrm{g} / \mathrm{L}$ & 2,5 & 75 & 250 & Diviso per fasce d'età \\
\hline PTH & $\mathrm{pmol} / \mathrm{L}$ & & & & $\mathrm{ng} / \mathrm{L}$ & 9,43 & 1,6 & 6,9 & \\
\hline BNP/NTproBNP & $\mathrm{ng} / \mathrm{L}$ & & & & $\mathrm{pg} / \mathrm{mL}$ & & & $<450$ & Diviso in 2 fasce d'età \\
\hline Troponina I & $\mathrm{ng} / \mathrm{L}$ & & & & & & & & \\
\hline Troponina $\mathrm{T}$ & $\mathrm{ng} / \mathrm{L}$ & & & & & & & $<50$ & Diviso per fasce d'età \\
\hline Calcio Urine $24 \mathrm{~h}$ & $\mathrm{mmol} / 24 \mathrm{~h}$ & 2,5 & 7,5 & & $\mathrm{mg} / 24 \mathrm{~h}$ & 40,1 & 2,5 & 8 & \\
\hline Urato Urine $24 \mathrm{~h}$ & $\mathrm{mmol} / 24 \mathrm{~h}$ & 1,5 & 4,5 & & $\mathrm{mg} / 24 \mathrm{~h}$ & 595 & 1,2 & 6 & \\
\hline Fosfato Urine $24 \mathrm{~h}$ & $\mathrm{mmol} / 24 \mathrm{~h}$ & 15 & 50 & & $\mathrm{~g} / 24 \mathrm{~h}$ & 31 & 12 & 40 & \\
\hline Magnesio Urine $24 \mathrm{~h}$ & $\mathrm{mmol} / 24 \mathrm{~h}$ & 2,4 & 6 & & $\mathrm{mg} / 24 \mathrm{~h}$ & 24,3 & 2,5 & 8,6 & \\
\hline
\end{tabular}

Intanto, in quegli stessi anni, si stava sviluppando nel Regno Unito il programma Acting for change. Si tratta di un progetto molto ambizioso di rinnovamento della Medicina di Laboratorio. Il Department of Health ha attivato nel biennio 2005-2006, nell'ambito del Modernising Pathology Programme, un programma formativo nazionale per consentire a quanti lavorano nei servizi di medicina di laboratori del NHS di concentrasi sui problemi più importanti, a livello sia generale sia locale. L'Action learning è concepito per aiutare gli operatori a migliorare il proprio servizio e l'intera organizzazione. La premessa è che si impara meglio quando si pianificano le soluzioni di problemi reali e concreti in piccoli gruppi, denominati action learning sets. Gli argomenti selezionati nel 2005 sono stati: lo sviluppo del personale, la standardizzazione, i network, la medicina primaria e la sicurezza dell'identificazione dei pazienti mediante "braccialetti". La standardizzazione degli intervalli di riferimento è stata affrontata dal Learning set di North Trent e il risultato del lavoro è 
Tabella 2 Intervalli di riferimento proposti dal programma Pathology Harmonization e intervalli usati dal Laboratorio Unico di AVR

\begin{tabular}{|c|c|c|c|c|c|c|c|c|c|}
\hline \multirow[b]{2}{*}{ Analita } & \multicolumn{4}{|c|}{$\begin{array}{c}\text { Intervalli di riferimento proposti } \\
\text { da Pathology Harmonization (pediatrici) }\end{array}$} & \multicolumn{5}{|c|}{ Intervalli di riferimento usati in AVR } \\
\hline & U.M. & inf. & sup. & note & U.M. & fatt. conv. & inf. & sup. & note \\
\hline Sodio & $\mathrm{mmol} / \mathrm{L}$ & 133 & 146 & & & & & & \\
\hline Potassio & $\begin{array}{l}\mathrm{mmol} / \mathrm{L} \\
\mathrm{mmol} / \mathrm{L} \\
\mathrm{mmol} / \mathrm{L}\end{array}$ & $\begin{array}{l}3,4 \\
3,5 \\
3,5\end{array}$ & $\begin{array}{r}6 \\
5,7 \\
5\end{array}$ & $\begin{array}{l}\text { Neonato } \\
<1 \text { anno } \\
1-16 \text { aa }\end{array}$ & & & & & \\
\hline Urea & $\begin{array}{l}\mathrm{mmol} / \mathrm{L} \\
\mathrm{mmol} / \mathrm{L} \\
\mathrm{mmol} / \mathrm{L}\end{array}$ & $\begin{array}{r}0,8 \\
1 \\
2,5\end{array}$ & $\begin{array}{l}5,5 \\
5,5 \\
6,5\end{array}$ & $\begin{array}{l}\text { Neonato } \\
<1 \text { anno } \\
1-16 \text { aa }\end{array}$ & & & & & \\
\hline Magnesio & $\begin{array}{l}\mathrm{mmol} / \mathrm{L} \\
\mathrm{mmol} / \mathrm{L}\end{array}$ & $\begin{array}{l}0,6 \\
0,7\end{array}$ & $\begin{array}{l}1 \\
1\end{array}$ & $\begin{array}{l}\text { Neonato } \\
1-16 \text { aa }\end{array}$ & & & & & \\
\hline Lattato & $\mathrm{mmol} / \mathrm{L}$ & 0,6 & 2,5 & & & & 0,5 & 2,2 & \\
\hline Bilirubina & $\mu \mathrm{mol} / \mathrm{L}$ & & $<21$ & $1-16$ aa & & & & & \\
\hline Albumina & $\begin{array}{l}\mathrm{g} / \mathrm{L} \\
\mathrm{g} / \mathrm{L} \\
\mathrm{g} / \mathrm{L}\end{array}$ & $\begin{array}{l}30 \\
30 \\
30\end{array}$ & $\begin{array}{l}45 \\
45 \\
50\end{array}$ & $\begin{array}{l}\text { Neonato } \\
<1 \text { anno } \\
1-16 \text { aa }\end{array}$ & & & & & \\
\hline Calcio & $\begin{array}{l}\mathrm{mmol} / \mathrm{L} \\
\mathrm{mmol} / \mathrm{L}\end{array}$ & $\begin{array}{r}2 \\
2,2\end{array}$ & $\begin{array}{l}2,7 \\
2,7\end{array}$ & $\begin{array}{l}\text { Neonato } \\
1-16 \text { aa }\end{array}$ & & & & & \\
\hline Fosfato & $\begin{array}{l}\mathrm{mmol} / \mathrm{L} \\
\mathrm{mmol} / \mathrm{L} \\
\mathrm{mmol} / \mathrm{L}\end{array}$ & $\begin{array}{l}1,3 \\
1,3 \\
0,9\end{array}$ & $\begin{array}{l}2,6 \\
2,4 \\
1,8\end{array}$ & $\begin{array}{l}\text { Neonato } \\
<1 \text { anno } \\
1-16 \text { aa }\end{array}$ & $\begin{array}{l}\mathrm{mg} / \mathrm{dL} \\
\mathrm{mg} / \mathrm{dL} \\
\mathrm{mg} / \mathrm{dL}\end{array}$ & $\begin{array}{l}3,1 \\
3,1 \\
3,1\end{array}$ & $\begin{array}{l}1 \\
1 \\
1\end{array}$ & $\begin{array}{l}2,6 \\
2,2 \\
1,8\end{array}$ & \\
\hline Fosfatasi Alcalina (ALP) & $\begin{array}{l}\mathrm{U} / \mathrm{L} \\
\mathrm{U} / \mathrm{L}\end{array}$ & $\begin{array}{l}70 \\
60\end{array}$ & $\begin{array}{l}380 \\
425\end{array}$ & $\begin{array}{l}\text { Neonato } \\
1-16 \text { aa }\end{array}$ & & & & $\begin{array}{l}<250 \\
<300\end{array}$ & \\
\hline Ammonio & $\begin{array}{c}\mu \mathrm{mol} / \mathrm{L} \\
\mu \mathrm{mol} / \mathrm{L} \\
\mu \mathrm{mol} / \mathrm{L}\end{array}$ & & $\begin{array}{r}<150 \\
<100 \\
<50\end{array}$ & $\begin{array}{l}\text { prematuro } \\
\text { Neonato } \\
1-16 \text { aa }\end{array}$ & & & & & \\
\hline
\end{tabular}

stato una standardizzazione di strumenti e metodi per la determinazione di elettroliti ed esami di funzionalità epatica [14]. Il successo dell'iniziativa ha portato a ripeterla nel triennio 2006-2008: i topici si sono ampliati e il lavoro è diventato più sistematico: costi, gestione delle richieste, armonizzazione degli intervalli di riferimento, consolidamento dei servizi di immunologia, comunicazione tra laboratori, "Lean, six-sigma", richiesta degli esami e invio del referto in medicina primaria, servizio di pronta disponibilità, servizio di monitoraggio terapia anticoagulante, Point of care testing, riorganizzazione del servizio di medicina di laboratorio, trasporti [15]. In questa occasione il Learning set è stato quello di West Midland. È diventato anche più esplicito che l'obiettivo era quello di estendere il progetto a livello nazionale. In effetti, nel novembre 2008 si è svolta la riunione in cui laboratoristi di Inghilterra, Galles, Scozia e Irlanda del Nord hanno approvato un primo pacchetto di proposte "armonizzate" e, soprattutto, definito il metodo per armonizzare l'unità di misura per un analita [16]. Il percorso seguito per valutare se si poteva armonizzare l'intervallo del sodio è stato, per esempio, il seguente:

1. nella regione del West Midland gli intervalli erano molto diversi e andavano da 138-146 $\mathrm{mmol} / \mathrm{L}$ a $130-155 \mathrm{mmol} / \mathrm{L}$;
2. è stato diffuso un questionario a tutti i laboratori dell'area per avere informazioni sull'intervallo e sull'analizzatore impiegato;

3. una riunione successiva ha consentito di verificare che le differenze non fossero collegate a differenze di metodo e strumento o a studi sulla popolazione locale che dimostravano la necessità di adottare un intervallo "personalizzato";

4. poiché le differenze erano legate semplicemente a cause storiche, si è raggiunta la conclusione di adottare come intervallo di riferimento: $133-146 \mathrm{mmol} / \mathrm{L}$.

Per altri analiti, tipicamente gli ormoni e i marcatori tumorali, le differenze tra metodi e strumenti erano talmente rilevanti da non consentire un' armonizzazione che sarà possibile solo quando sistemi analitici diversi daranno risultati più omogenei.

Il Pathology Harmony Group ha esteso la sua attività e nel gennaio 2011 Julian Barth, Presidente dell'Association for Clinical Biochemistry, James Kenneth Rae, Presidente dell'Institute of Biomedical Science e Danielle Freedman, Presidente del SAC Clinical Biochemisry del Royal College of Pathologists, hanno raccomandato l'introduzione di intervalli di riferimento armonizzati per alcuni analiti nell'adulto e nel bambino (Tabb. 1, 2). Al momento non sono stati presentati intervalli per l'Ematologia e per l'Immunologia, ma il lavoro è in corso anche in questi ambiti. 


\section{Conclusioni}

Sembra che, anche se lo standard C28-A3 dedica poco spazio agli intervalli di riferimento armonizzati o comuni, le esigenze informatiche, cliniche e amministrative stiano spingendo sempre più in questa direzione. L'esperienza scandinava è difficilmente esportabile al di fuori di quel contesto; in quella realtà i numeri sono piccoli, la tradizione nella standardizzazione è notevole, l'autorevolezza della disciplina è probabilmente più grande di quella goduta in altri paesi.

L'esperienza progressiva di Australia, Nuova Zelanda e Regno Unito sembra invece più praticabile in tempi brevi e, d'altra parte, non fa altro che riprendere iniziative che nel nostro Paese sono state condotte a partire dagli anni ottanta [8, 17, 18].

Riteniamo sia venuto il momento di intraprendere, in modo pragmatico, prudente ma determinato, un percorso per l'introduzione di Intervalli di riferimento comuni in tutti quei casi in cui questo rappresenta la soluzione forse non perfetta, ma sicuramente più efficace. $\mathrm{Ci}$ auguriamo, pertanto, che, prima a livello regionale e poi nazionale, si valuti lo stato dell'arte raggiunto da NORIP e da Pathology Harmony, e si attivino percorsi analoghi nel nostro Paese con le correzioni e le personalizzazioni necessarie. Va considerato che il consolidamento dell'attività di medicina di laboratorio facilita notevolmente queste dinamiche. Oggi, anche nel nostro Paese, sono diverse le realtà in cui ambiti regionali o semiregionali sono serviti dallo stesso laboratorio, ovvero da laboratori che impiegano uguale strumentazione, uguali metodi e uguali unità di misura [19, 20].

Conflitto di interessi: Nessuno

\section{Bibliografia}

1. Ceriotti F, Hinzmann R, Panteghini M (2009) Reference intervals: the way forward. Ann Clin Biochem 46:8-17

2. Ceriotti F (2007) Gli intervalli di riferimento nel nuovo millennio. Biochimica Clinica 31: 254-266

3. Clinical and Laboratory Standards Institute (CLSI) (2008) Defining, Establishing, and Verifying Reference Intervals in the
Clinical Laboratory; Approved Guideline - Third Edition. CLSI Wayne, Pennsylvania, USA

4. Schneider AJ (1960) Some thoughts on normal, or standard, values in clinical medicine. Pediatrics 26:973-984

5. Beckett G, MacKenzie F (2007) Thyroid guidelines - are thyroidstimulating hormone assays fit for purpose? Ann Clin Biochem 44:203-208

6. Andrew CE, Hanning I, McBain AM et al (2000) A model for a multicentre approach to the derivation of reference intervals for thyroid hormones and testosterone for laboratories using identical analysers. Clin Chem Lab Med 38:1013-1019

7. Friedberg RC, Souers R, Wagar EA et al (2007) The Origin of Reference Intervals. A College of American Pathologists QProbes Study of "Normal Ranges" Used in 163 Clinical Laboratories. Arch Pathol Lab Med 131: 348-357

8. Andreotti A, Bagni B, Baraghini GF et al (eds) (1991) Le analisi di laboratorio. Artestampa, Modena

9. Rustad P, Felding F, Franzson L et al (2004) The Nordic Reference Interval Project 2000: recommended reference intervals for 25 common biochemical properties. Scand J Clin Lab Invest 64:271-284

10. NORIP http://www.furst.no/norip. Data di consultazione: 8 Marzo 2011

11. Rustad P (2010) The Nordic Reference Interval Project 2000. Concept and consequences. http://www.furst.no/norip/info/ NORIP_foredrag.pdf. Data di consultazione: 8 Marzo 2011

12. Jones GR, Barker A, Tate J et al (2004) The case for Common Reference intervals. Clin Biochem Rev 25:99-104

13. Jones GR, Barker A (2007) Standardisation of reference intervals: an Australasian view. Clin Biochem Rev 28:169-173

14. NHS (2007) Modernising from within: action learning solutions for pathology. http://www.dh.gov.uk/prod_consum_dh/groups/ dh_digitalassets/@dh/@en/documents/digitalasset/dh_074707.p df. Data di consultazione: 8 Marzo 2011

15. NHS (2008) Acting for change: transforming pathology services through action learning. http://www.dh.gov.uk/en/Publications andstatistics/Publications/PublicationsPolicyAndGuidance/DH_ 090079. Data di consultazione: 8 Marzo 2011

16. http://www.pathologyharmony.co.uk. Data di consultazione: 8 Marzo 2011

17. Zardo L, Secchiero S, Sciacovelli L et al (1999) Reference intervals: are interlaboratory differences appropriate? Clin Chem Lab Med 37:1131-1133

18. Sciacovelli L, Zardo L, Secchiero S et al (2003) Interpretative comments and reference ranges in EQA programs as a tool for improving laboratory appropriateness and effectiveness Clin Chim Acta 333:209-219

19. Dorizzi RM, Nizzoli M (2009) Gli intervalli di riferimento in Endocrinologia; il caso del TSH. RIMeL/IJLaM 5 (3-S1):52-61

20. Dorizzi RM, Maltoni P (2010) Ruolo del Laboratorio nello studio della patologia surrenalica: logiche di intervento e strumenti di intervento. RIMeL/IJLaM 6:237-47 